\title{
The primate circadian rhythm during isolation'
}

\author{
DEPARTMENT OF NEUROLOGY AND PSYCHIATRY, HENRY FORD HOSPITAL
}

The present study presents the measurement of the primate circadian rhythm as it occurs in constant environmental conditions during a 90-day period of isolation. The major findings indicate that the recorded physiological and behavioral activity followed a $23-\mathrm{hr}$. periodicity when stimulus change was minimized.

Classically, research has been concerned with activity as an independent drive and/or with how it is affected by external and internal stimuli (see Hall, 1961; Harker, 1964). While there can be little argument that any specific, overt behavior requires an active organism and that research has defined some of the important conditions which influence activity, it is equally true that no author has posited the view that activity can continue indefinitely even under the most optimal of conditions. This implicit acceptance of a limited influence of external stimulus conditions would then appear to emphasize endogenic factors as the ultimate determinants of an organism's activity such that specification of the circadian rhythm as it occurs under constant environmental conditions should provide a contribution to understanding the "when" of behavior. However, while circadian measurement has been attempted with several vertebrate and lower species, little work has been done with the subhuman primate so that the present research has attempted, under constant environmental conditions, to specify the cyclic nature of activity as indexed by both physiological (pulse rate) and overt (bar press) criteria.

Subject

The $S$ for this study was an adult Nemestrina monkey from the colony maintained at the Edsel B. Ford Institute for Medical Research.

Apparafus

The apparatus for this experiment consisted of an acoustical cubical (Industrial Acoustical Co., Inc., Model No. 403) with inside floor dimensions of 84 in. by 84 in., and a wall height of 78 in. Within this area, and centered against the wall opposite the entrance door, was the monkey compartment ( $3 \mathrm{ft}$. wide by 26 in. deep by 78 in. high) constructed of double-walled acoustical material. The remaining area of the cubicle was used as an anteroom to house the equipment necessary to maintain the monkey undisturbed for the 90day experimental period.

Within the monkey compartment, the $S$ was restrained in a primate chair situated directly in front of a response panel. The response panel contained a single lever, reward receptacle, speaker and the forward end of a food conveyor belt. Light within the chamber was provided by two 100-W, 115-V bulbs wired in series and located approximately 1-1/2 ft. above the S's head. A drinking tube was mounted on the primate chair directly to the animal's right. Other allied equipment consisted of a commercially available random-noise generator and amplifier along with necessary plug-in timer and relay panels, all of which were located outside the acoustical chamber. The S's bar-press activity was recorded on a cumulative event recorder.

Waste products were flushed from the monkey compartment into a container in the anteroom by an automatic recirculating water system connected to a drop pan mounted under the primate chair. This system operated on the average of six times per day from a water reservoir located outside the acoustical chamber. Bacterial growth in the reservoir and associated tubing was checked by changing the water in the external reservoir twice daily and by the weekly addition of sodium azide at a concentration of $20 \mathrm{gm}$ per 5 gallons of water. Continuous random noise at a level of $75 \mathrm{~dB}$, the reference being .0002 dyne per $\mathrm{cm}$ sq., effectively masked both the operation of the flushing system and daily anteroom maintenance activities.

The temperature within the monkey chamber, recorded at approximately $0830 \mathrm{hr}$. daily, remained between $78^{\circ}$ and $87^{\circ} \mathrm{F}$ during the entire experiment with a mean difference between successive days' observations of $1.75^{\circ} \mathrm{F}$.

\section{Procedure}

Prior to the 90-day isolation, pulse leads were implanted under antiseptic conditions in the right and left abdominal regions of the $S$. These leads, when connected to a Grass electroencephalograph, provided a reasonable representation of the animal's pulse which was automatically monitored for $5 \mathrm{~min}$. out of every $30 \mathrm{~min}$. throughout the experiment.

During the experimental period, food (monkey chow pellets) and water were provided ad lib while FR behavior was "rewarded" with $190 \mathrm{mg}$ banana-flavored pellets. However, the pre-isolation training ratio of 100 responses per pellet proved too long under the experimental conditions so that on day 32 the ratio was dropped to 25 with a subsequent increase (day 39) to 50, where it remained until termination of the experiment.

\section{Resulis}

Prior to discussing the particular results, it should be noted that the animal was examined immediately following its removal from the chamber and during the subsequent week by the project veterinarian. At each of these examinations, there were no outward indications of any deleterious effects from the 90-day period of isolation and restraint except for a hyperreactivity during the initial examination which dissipated soon after the animal was returned to the colony. 


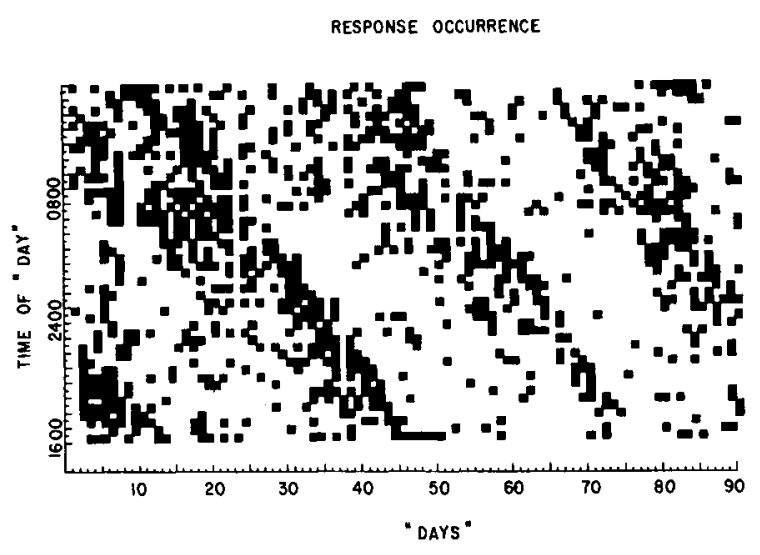

Fig. 1. Response emission during the individual $1 / 2-\mathrm{hr}$. periods of each of the $\mathbf{9 0}$ days of isolation. See text for complete explanation.

The average daily food intake (monkey chow pellets) during the 90-day period was 37.7 pellets per day with a standard deviation of 9.75 pellets. It must be noted, however, that there was a significant decrease (t-test, $p<.001)$ in pellets eaten during the terminal 30 days of the experiment as compared to the quantity eaten during the initial 30 days. The water consumption data, unfortunately, cannot be so specified due to a variable amount of dripping from the tube after the $S$ drank.

The major findings of the present study are presented in Figs. 1 and 2 which respectively present the pattern of response occurrences and upper quartile pulse rates over isolation "days," where a "day" commenced at $1600 \mathrm{hr}$. and terminated at $1559 \mathrm{hr}$. Both figures are organized on identical formats and present the total experimental period as 4,320 individual cells. A particular cell is darkened if, for Fig. 1, one or more responses occurred during the $1 / 2-\mathrm{hr}$. period represented by the cell in question or if, considering Fig. 2, the pulse rate for that $1 / 2-\mathrm{hr}$. interval was

UPPER GUARTILE PULSE RATES

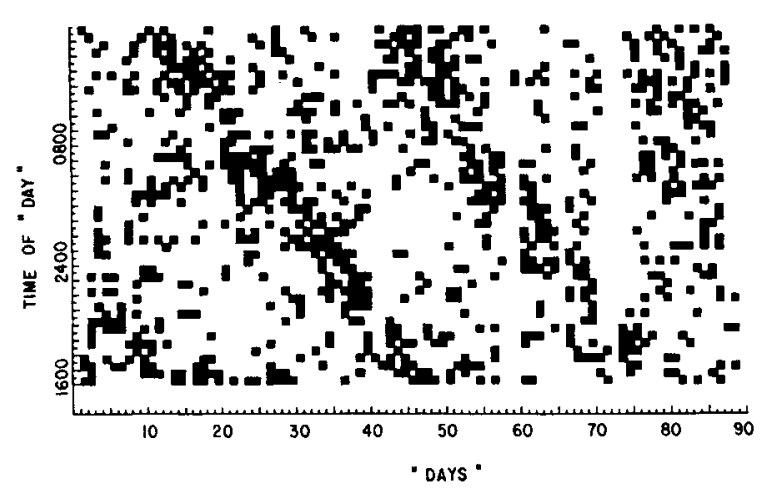

Fig. 2. Occurrences of upper quartile pulse rates monitored for a duration of $5 \mathrm{~min}$. each $1 / 2-h r$. Note: those days which contain less than 12 darkened cells are chieny the result of dried ink in the writing pen. in the upper quartile range. The figures thus respectively present a behavioral and a physiological index of activity within an experimental "day" and more importantly how the period of activity varies across days when external stimulus change is minimized.

\section{Discussion}

The results from the procedures described demonstrate that the Nemestrina circadian rhythm, as measured by response emission and pulse rate, does not correspond to the clock day of $24 \mathrm{hr}$. but is shorter by approximately $1 \mathrm{hr}$. While exact specification of the endogenic rhythm is difficult, that the periodicity is faster than $24 \mathrm{hr}$. is clear from the slope of patterns presented in Figs. 1 and 2 while the validity of the chosen measures is demonstrated by their consistency and similarity over the experimental period. It is possible, however, to argue that the measures are not independent estimates of a circadian process but rather that a cause and effect relation exists between responding and increased pulse rate. However, this possibility is all but precluded by the temporal arrangement of our pulse monitoring (the last $5 \mathrm{~min}$. of every $1 / 2 \mathrm{hr}$.) and the fact that we are measuring response emission (one or more bar presses) which, during the initial 31 days of the experiment, very nearly dropped to zero. Thus, in the present situation, responding would necessarily have had to occur at a high rate, in approximately the final $10 \mathrm{~min}$. of every $1 / 2-\mathrm{hr}$. interval to effect the correlation observed and this was definitely not the case. ${ }^{2}$ We thus feel that the two measures are without a cause and effect relation but are simply in close agreement that the periodicity of the underlying endogenic process is faster than what is usually considered the diurnal rhythm of monophasic organisms.

Finally, the results tend to support arguments for the stimulus control of an organism's activity in unaltered environmental conditions (see Bruce, 1960). However, the extent of this control or entrainability of the endogenically determined activity remains an open question as definitive studies have yet to be performed; so that while the primate is normally considered a diurnal beast, the extent and range of its daily activity as precisely quantified over extended periods of observation are not known with any certainty.

\section{References}

Bruce, V. G. Environmental entrainment of circadian rhythms. Cold Spring Harbor Symp. Quant. Biol., 1960, 25, 29-48.

Hall, J. F. Psychology of motivation. New York: J. B. Lippincott Co., 1961.

Harker, J. E. The physiology of diurnal thythms. Cambridge, Mass.: Cambridge University Press, 1964.

\section{Notes}

1. The author wishes to acknowledge the support of grant NASr-83 from the National Aeronautic and Space Administration.

2. Individual daily records are on file at Henry Ford Hospital, Detroit, Michigan. 